\title{
Greenness of things
}

\author{
Rajender S. Varma ${ }^{1}$ (1)
}

Published online: 4 September 2021

(c) The Author(s), under exclusive licence to Springer-Verlag GmbH Germany, part of Springer Nature 2021

Green chemistry is now nearly three decades old since the term was coined with laying down of 12 principles that envisioned minimum risk as the performance measure while creating newer chemical methods; relatively cleaner processes are emphasized as the alternatives to conventional chemical syntheses and transformations are being explored. A closely connected and often misused term, sustainability, has attained the status of a priority agenda for private companies and governments worldwide. The varied nature of the chemical synthesis domain necessitates assorted greener routes in our search to attain sustainability. However, it should be noted that 'greener' chemistry was practiced without the use of that specific term, an ancient example being the 1905 Nobel Chemistry Prize winning solventless synthesis of indigo by Adolf von Baeyer who single-handedly eliminated its access via the cultivation in fields; numerous improvements to secure the catalytic synthesis of desired isomers in chemical syntheses are yet another significant contribution over the last century. Several publishing houses have established numerous outlets ${ }^{1}$ for dissemination of details pertaining to the journey on green and sustainable pathways (Varma, 2014) on varied topics including the use of renewable carbonaceous materials (Varma, 2019).

Despite the 12 misunderstanding of Green Chemistry, there have been several yardsticks that have been established to define the greenness of the protocols including the development of matrices and environmental (E)-factor (ratio of the mass of waste per mass of product) introduced originally in 1992 by Sheldon and its update after 25 years. A major thrust area for accomplishing this objective is to explore alternative efficient reaction conditions and especially in eco-friendly media (solvents) to complete the preferred chemical conversions with minimum generation of waste or by-products and without the use of traditional volatile

Rajender S. Varma

Varma.Rajender@epa.gov

1 Regional Center of Advanced Technologies and Materials, Czech Advanced Technology and Research Institute, Palacky University, Šlechtitelů 27, 78371 Olomouc, Czech Republic organic solvents (VOCs), where feasible. Therefore, several innovative approaches have emerged, namely reactions via mechanochemical mixing (grinding), usage of solvent-free (dry media) conditions (Varma 1999) with or without solidsupported reagents and the alternate activation methods that exploit ultrasonic irradiation (Chatel and Varma, 2019) and microwave (MW) irradiation (Martina et al. 2021) for faster syntheses. These systems have circumvented some of the drawbacks connected to extravagant heating; ball-milling, a solid-state size diminution protocol that could be easily adapted for large-scale appliances as attested by its use in pharma and paint industry, is a good example. Mechanochemical handling of organic/inorganic molecules under solvent-free or bare minimal solvent usage precludes the use of toxic metals and solvents and is garnering special attention. Whereas MW irradiation offers the selective heating of polar entities (volumetric core), sonochemistry augments the mass transfer and chemical reaction via the process of acoustic cavitation (Chatel and Varma, 2019). The selective engagement of microwaves by polar mediators and molecules in a multiphase system could likewise replace as a phase transfer catalyst without the deployment of actual phase transfer reagent, thus facilitating the noted increase of rate as has been observed for ultrasonic irradiation. Experimental observations and recent advancements in measurements and equipment for numerous reaction systems have been consistent with the mechanistic postulation wherein the polar transition state of the reaction is favored by MW irradiation with respect to the dielectric polarization nature of MW energy transfer (Martina et al. 2021).

The perspective/review in this issue of Clean Technology and Environmental Policy (Winterton 2021) provides an extensive commentary on the "green solvents", a concept that has produced a sizeable chemical literature including the growth of purported 'neoteric' solvents. However, a

\footnotetext{
${ }^{1}$ Leading journals on these themes include: Green Chemistry (RSC), ACS Sustainable Chemistry \& Engineering (ACS), ChemSusChem (Wiley) and Current Opinion on Green and Sustainable Chemistry (Elsevier) among others such as Sustainable Chemistry and Pharmacy (Elsevier) and Clean Technology and Environmental Policy (Springer).
} 
judicious synopsis of proclaimed data ascertains that only handful of novel materials have yet found pervasive use as solvents. An additional emphasis on the use of process intensification to generate solvents such as ionic liquids (ILs) using organic solvents would have been even more praiseworthy, after all, you cannot make a greener solvent using another solvent even without paying detailed attention to life cycle assessment (LCA). Additionally, solventfree strategy needs to be highlighted as such technologies embracing mechanochemical means to accomplish chemical reactions would support the adage, 'no solvent is good solvent'. Broader sustainability queries, especially espousing the exploitation of non-fossil sources to produce solvents, are more important than inherent 'greenness'. The appraisal of the inclusive creation of solvents or chemicals from biomass, in sustainable terms, is of paramount significance besides the ready accessibility of such resources; raw materials explored for solvent procurement or generation of useful chemical entities are not feasible if they are presently being utilized for consumption by animals or humans. Common agricultural residues like the wheat, rice or corn straw, a common feed for animals, is already a depleted resource with the increased meat-eating habits in overcrowded countries of Asia. The economic viability is indispensable for the ultimate adaptation and execution of marketable manufacturing developments. Thus, it is essential that all the ensuing constituents secured with utmost conversion from biomass valorization are fully utilized in its entirety for high-value functions (Varma 2019).

Furthermore, the land use and associated capital and shipping costs, and the gathering of bulky biomass supplies, is an energy- and labor-demanding enterprise. Thus, it would be prudent to have the deployment of modular handling appliances in the fields close to materials resource where primary treatment and bulk diminution can be undertaken. Preferably, leftover agricultural scraps and thrown away food and fodder should be the ideal choice which can take advantage of the abundant solar energy or visible light in the fields.

Lignocellulosic materials from the woody plants and climbers, being encountered in common sight, have been widely explored during the last decades. Nevertheless, almost, two thirds of our Biosphere are water bodies housing abundant sea creatures which adorn a very useful biopolymer, Chitin. Instead of making use of this prized, renewable, and plentiful resource, tipping fees are demanded to dispose this valued material into scarcely available landfills. It is strange that we fastidiously investigate newer nitrogenaugmented carbonaceous matters by doping various carbon forms namely graphene or search means to produce graphitic carbon nitrides and yet disregard bountiful nitrogen-rich supply from the Nature in the form of chitosan and chitin. Furthermore, the growing algae and resulting overabundance of produces from sea water is an alluring suggestion for future investigations as the progress perseveres with novel catalytic protocols for biomass-derived chemical entities rather than pursuing traditional fossil-fuel originated chemicals.

The deployment of earth-abundant and safer catalysts under ambient and solvent-free conditions and innovative process intensification utilizing continuous flow circumstances is essential (Martina et al. 2021). Innovative advancements on these topics, principally encompassing benign reaction media namely polyethylene glycol (PEG), water and in combination with ultrasonic and MW irradiation and/or ball-milling under solvent-free conditions, may help attain sustainable means for chemical conversion and synthesis. Remarkably, even the production of emerging nano-based materials from abundant and renewable resources (Varma 2012) is feasible with high-value appliances in diverse disciplines (Iravani and Varma 2019, 2020, 2021).

\section{References}

Chatel G, Varma RS (2019) Ultrasound and Microwave Irradiation: Contributions of Alternate Physicochemical Activation Methods to Green Chemistry. Green Chem 21:6043-6050. https://doi.org/ $10.1039 / \mathrm{c} 9 \mathrm{gc} 02534 \mathrm{k}$

Iravani S, R. S. Varma RS (2019) Plants and Plant-Based Polymers as Scaffolds for Tissue Engineering. Green Chem 21, 4839-4857; (2020) Green Synthesis, Biomedical and Biotechnological Applications of Carbon and Graphene Quantum Dots. A Review. Environ Chem Lett 18, 703-727; (2021) Plant Pollen Grains: A Move Towards Green Drug and Vaccine Delivery Systems. Nano-Micro Lett 13, 128.

Martina, K, Cravotto G and Varma RS (2021) Impact of Microwaves on Organic Synthesis and Strategies toward Flow Processes and Scaling Up. J Org Chem 86: (2021). https://doi.org/10.1021/acs. joc. $1 \mathrm{c} 00865$

Varma RS (1999) Solvent-free Organic Syntheses Using Supported Reagents and Microwave Irradiation. Green Chem 1:43-55

Varma RS (2012) Greener Approach to Nanomaterials and Their Sustainable Applications. Curr Opin Chem Eng 1:123-128

Varma RS (2014) Journey on Greener Pathways: From the use of Alternate Energy Inputs and Benign Reaction Media to Sustainable Applications of Nano-Catalysts in Synthesis and Environmental Remediation. Green Chem 16:2027-2041

Varma RS (2019) Biomass-derived Renewable Carbonaceous Materials for Sustainable Chemical and Environmental Applications. ACS Sustain Chem \& Eng 7:6458-6470

Winterton N (2021) The Green Solvent: A Critical Perspective. Clean Technologies and Environmental Policy. https://doi.org/10.1007/ s10098-021-02188-8

Publisher's Note Springer Nature remains neutral with regard to jurisdictional claims in published maps and institutional affiliations. 